\title{
DeepNeuron: an open deep learning toolbox for neuron tracing
}

\author{
Zhi Zhou ${ }^{1,2}$, Hsien-Chi Kuo $^{1}$, Hanchuan Peng ${ }^{1,2^{*}}$ and Fuhui Long ${ }^{1 *}$
}

\begin{abstract}
Reconstructing three-dimensional (3D) morphology of neurons is essential for understanding brain structures and functions. Over the past decades, a number of neuron tracing tools including manual, semiautomatic, and fully automatic approaches have been developed to extract and analyze 3D neuronal structures. Nevertheless, most of them were developed based on coding certain rules to extract and connect structural components of a neuron, showing limited performance on complicated neuron morphology. Recently, deep learning outperforms many other machine learning methods in a wide range of image analysis and computer vision tasks. Here we developed a new Open Source toolbox, DeepNeuron, which uses deep learning networks to learn features and rules from data and trace neuron morphology in light microscopy images. DeepNeuron provides a family of modules to solve basic yet challenging problems in neuron tracing. These problems include but not limited to: (1) detecting neuron signal under different image conditions, (2) connecting neuronal signals into tree(s), (3) pruning and refining tree morphology, (4) quantifying the quality of morphology, and (5) classifying dendrites and axons in real time. We have tested DeepNeuron using light microscopy images including bright-field and confocal images of human and mouse brain, on which DeepNeuron demonstrates robustness and accuracy in neuron tracing.
\end{abstract}

Keywords: DeepNeuron, Deep learning, Neuron tracing, Neuron morphology

\section{Introduction}

Over the past few decades, researchers have developed algorithms and tools to reconstruct (trace) 3D neuron morphology. A number of manual/semiautomatic neuron tracing software packages in both the public domain and commercial world have been developed [1-9]. To further promote the development of neuron tracing tools, the DIADEM challenge [10] and the BigNeuron project [11] were launched to compare different automated algorithms. At small or medium scales, many algorithms (base tracers) have been shown to produce meaningful reconstructions on high-quality neuron images. For large-scale image datasets, UltraTracer [12] provides an extendible framework to scale up the capability of these base tracers. Despite these efforts on algorithm and tool development, it remains an open question on how to

\footnotetext{
*Correspondence: hanchuanp@alleninstitute.org; fuhuil@alleninstitute. org

${ }^{1}$ Allen Institute for Brain Science, Seattle, USA

Full list of author information is available at the end of the article
}

faithfully reconstruct neuron morphology from challenging image datasets that have medium to low qualities and contain very complex neuron morphology.

Starting from a cell body, a neuron tracing process usually follows dendrites and axons, eventually connecting all such neuron signal as a tree that represents the morphology of the neuron. In light microscopy images, dendrites typically show continuous signal, whereas axons are often hard to trace due to their punctuated appearance and large, complex arborization patterns ([8]; see for example the bright-field images of biocytin-labeled neurons in the Allen Cell Type Database [24]). In addition, the image quality varies a lot depending on sample preparation, imaging process, cell types, and the healthiness of neurons. For instance, neuron signal could be continuous in one image, but dim and broken in another. It is difficult to automatically extract all such neuron signal under different conditions.

Several important steps in neuron tracing can be formulated as a classification problem. For example, detection of neuron signal from background is essentially 
foreground-background classification. Reconstruction of the topology of a neuron via connecting neuron fragments can be treated as connection-separation classification. In this aspect, a few studies used traditional machine learning and recent deep learning [13] models to produce neuron morphology. For example, Gala et al. introduced an active learning model by combining different features to automatically trace neurites [14]. Chen et al. proposed a self-learning-based tracing approach, which did not require substantial human annotations [15]. Fakhry et al. [16] and Li et al. [17] used deep learning neural networks to segment electron and light microscopy neuron images. Despite these algorithmic efforts, none of these methods provides publicly available tools to use on external datasets.

Nowadays, deep learning methods outperform traditional methods in many pattern recognition and computer vision applications. We analyzed commonly used modules of neuron tracing/editing workflows in real applications, and concluded that an Open Source deep learning toolbox would help this growing field. Using deep learning neural networks as the classification models, we develop DeepNeuron, which provides several essential modules to neuron tracing. For automated tracing, DeepNeuron can be used as either a new tracing algorithm to reconstruct neurites from difficult neuron images, or an extra processing component to improve other tracing algorithms. DeepNeuron could also assist annotators in manual tracing. Supporting extendable functions as plugins, currently DeepNeuron contains five commonly used modules (Fig. 1):

- Neurite signal detection automatically identifies 3D dendritic and axonal signal from background.

- Neurite connection automatically connects local neurite signal to form neuronal trees.

- Smart pruning filters false positive and refines automated reconstruction results.

- Manual reconstruction evaluation evaluates manual reconstructions and provides quality scores.

- Classification of dendrites and axons automatically classifies neurite types during real-time annotation.

\section{Five modules}

\subsection{Neurite signal detection}

Due to difficulties in sample preparations and imaging, neurite signals often appear broken in a 3D image. It is hard to use any existing automated tracing algorithm to reconstruct 3D neuronal structures when this happens. Even for human annotators, locating these isolated axonal signals from the noisy background is a daunting work. To reliably detect neurite signals, we introduce the neurite signal detection module based on deep CNN to classify signal and background. This allows us to precisely detect neurite signals without any preprocessing steps applied on the original image. To speed up the detection

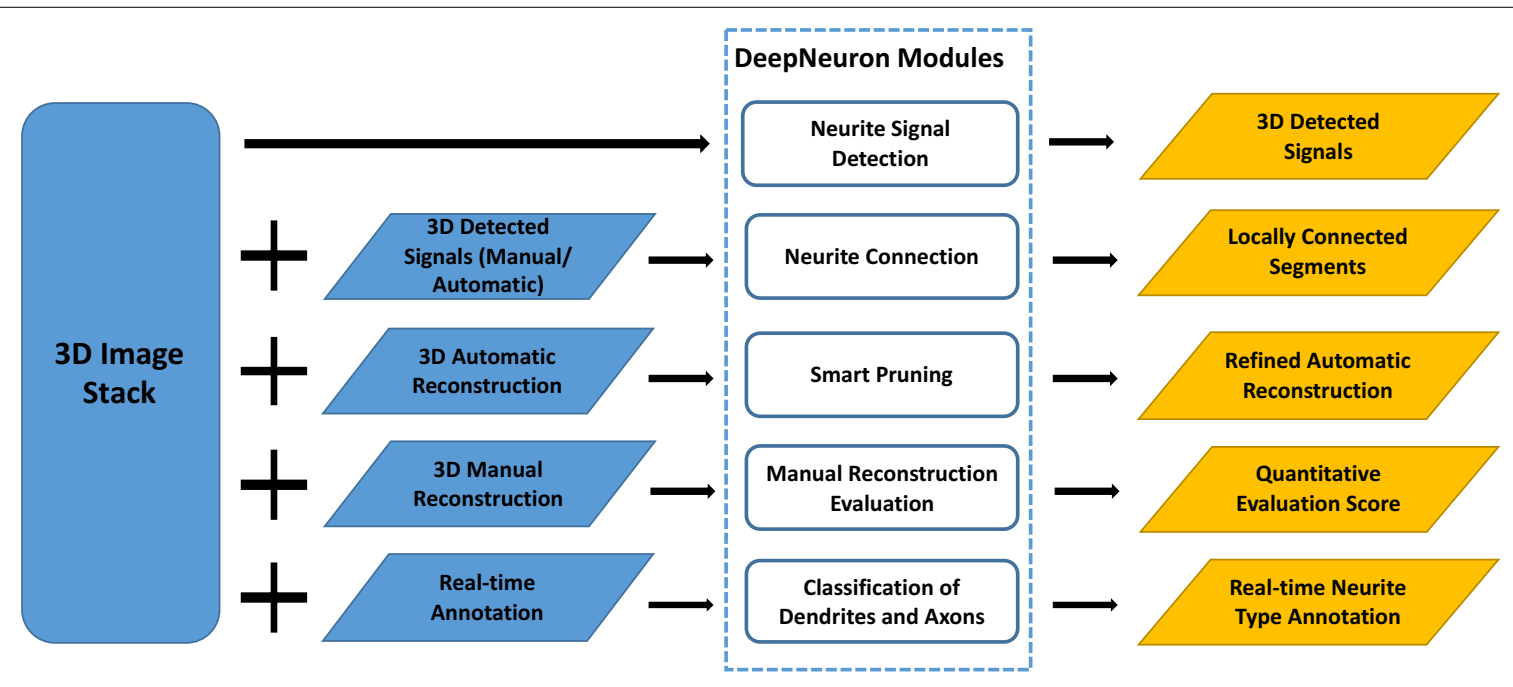

Fig. 1 The workflow of the Open Source DeepNeuron toolbox, which has five deep learning-based modules. Each DeepNeuron module has one or more processing components. Neurite signal detection module (Sect. 2.1) uses convolutional neural networks (CNNs) to do foreground/ background classification. Neurite connection module (Sect. 2.2) uses a revised Siamese network [21, 22] to connect neurite structure from detected neuron signals. Smart pruning module (Sect. 2.3) refines a neuron's morphology by using CNN models to filter out false positives. Manual reconstruction evaluation module (Sect. 2.4) uses the output of CNNs as quality scores to evaluate reconstructions. Finally, dendrites/axons classification module (Sect. 2.5) uses CNNs to perform multiclass classification to differentiate axons, dendrites, and background. Note that all the actual deep learning networks in our five modules can be replaced with other network models or user's own design 
and lower the GPU memory requirement, we used a twodimensional (2D) CNN model followed by 3D mapping to detect signal in 3D and achieved satisfactory results on our testing data. However, our framework is not limited to $2 \mathrm{D} \mathrm{CNN}$ but can also directly use $3 \mathrm{D} \mathrm{CNN}$ models (Fig. 2).

Manually reconstructed neurons were used as training samples. The 3D reconstruction of a neuron is represented as a tree, which contains a series of 3D X, Y, Z locations, radius, and topological "parent" of annotation nodes. To train the network, local 3D blocks (block size $61 \times 61 \times 61$ was used in our experiments) centered on manually annotated nodes in neurite segments were cropped from the original images. 2D maximum intensity projections (MIPs) of theses 3D blocks were used as the positive training set, and the same number of $2 \mathrm{D}$ background MIPs were randomly selected as the negative training set.

We tested our module using AlexNet [18] with five convolutional and three fully connected layers. Table 1 shows the fivefold cross-validation test of the module robustness. The training image dataset was partitioned into five equal size subsets $(1-24,25-48,49-72,73-96$, and $97-122$ as shown in Table 1). Four subsets were used for training, and the remaining single subset was used for

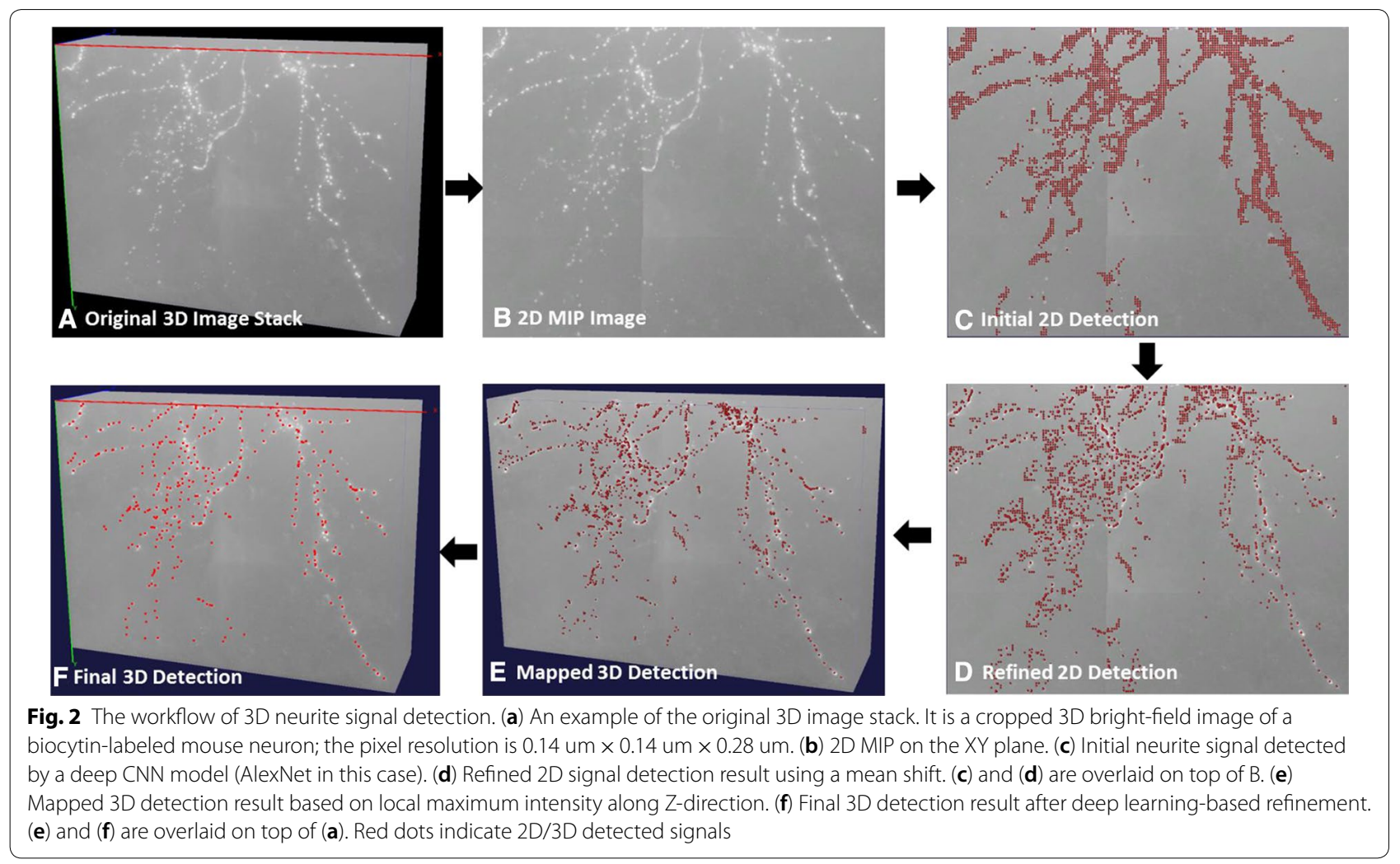

Table 1 Fivefold cross-validation on bright-field training sets

\begin{tabular}{|c|c|c|c|c|c|c|}
\hline \multirow[t]{2}{*}{ Training set } & \multicolumn{2}{|c|}{ Foreground accuracy } & \multicolumn{2}{|c|}{ Background accuracy } & \multicolumn{2}{|c|}{ Overall accuracy } \\
\hline & Training (\%) & Validation (\%) & Training (\%) & Validation (\%) & Training (\%) & Validation (\%) \\
\hline$\{1-122\} \backslash\{1-24\}$ & 98.77 & 97.78 & 98.97 & 96.87 & 98.87 & 97.33 \\
\hline$\{1-122\} \backslash\{25-48\}$ & 98.54 & 99.07 & 98.78 & 98.34 & 98.66 & 98.71 \\
\hline$\{1-122\} \backslash\{49-72\}$ & 98.67 & 98.28 & 98.78 & 99.13 & 98.73 & 98.71 \\
\hline$\{1-122\} \backslash\{73-96\}$ & 98.71 & 96.64 & 98.28 & 99.23 & 98.50 & 97.94 \\
\hline$\{1-122\} \backslash\{97-122\}$ & 98.64 & 99.02 & 98.77 & 98.41 & 98.71 & 98.72 \\
\hline Average & 98.67 & 98.08 & 98.83 & 98.44 & 98.75 & 98.26 \\
\hline
\end{tabular}


validation. Our results show our overall accuracy $>98 \%$ for both training and validation.

In testing, we first projected the original 3D image stack onto the XY plane and generated a MIP image. We then cropped $2 \mathrm{D}$ patches using a sliding window with $n$-pixel stride. These patches were classified into patches centered on foreground or background pixels using our trained CNN model. To further improve classification accuracy and exclude false positive patches, we applied mean shift [19] to the detected foreground patches and map them back to the actual 3D locations based on the local maximum intensity along Z. Finally, we classified these 3D detected signals using our CNN model again based on the MIPs of the local 3D blocks.

We applied our module to two challenging datasets of mouse neurons. The first set was a bright-field biocytin-labeled mouse neuron dataset from Allen Cell Type Database [24]. The second set was a whole mouse brain data imaged by fMOST imaging technology [20]. For the first dataset, we used 122 bright-field neuron image stacks and their associated manual reconstructions as the training set and produced $\sim 813 \mathrm{~K}$ training samples including $\sim 404 \mathrm{~K}$ foreground patches, and $408 \mathrm{~K}$ background patches. For the whole mouse brain dataset, we used $\sim 493 \mathrm{~K}$ training samples including $\sim 252 \mathrm{~K}$ foreground patches, and $\sim 241 \mathrm{~K}$ background patches from 22 whole mouse brain images. Figure 3 shows two examples of axon detection results. Using neurite signal detection module, most of axonal signals have been precisely detected in both datasets.

\subsection{Neurite connection}

A complete neuron forms a tree structure that is composed of continuous neurite segments. Global, local, and topological features including total length, bifurcations, terminal tips, and more others are used to study the neuronal morphology. These features have to be extracted from neurite segments instead of dots. Therefore, finding the continuity of neurites and connecting neurite segments are critical steps in neuron tracing. Generally, automated tracing algorithms can achieve good performance on connecting neurite segments with small gaps based on the continuity of segment orientations. However, it is difficult to automatically connect dots-like neurite signals. Using the spatial distance between these signals as the weight, minimal spanning tree (MST) provides a possible solution. However, without biological context, it could also introduce topological errors. Human beings are good at finding the continuity of isolated signals as per their observations and domain knowledge. By learning the neurite connectivity from a large dataset annotated by humans, a deep learning-based MST (DMST) approach we proposed can successfully connect neurite segments with relatively big gaps (Fig. 4).

Siamese networks $[21,22]$ are used among tasks that involve finding similarity or the relationship between two subjects being compared. Our revised Siamese model in this work includes two identical arms. Each consists of two convolutional layers with max pooling, followed by three fully connected layers. The two arms are then fed to a contrastive loss function to produce a binary decision.

In training (Fig. 4a), we used pairs of patches generated from two consecutive annotation nodes as positive training samples, and pairs of patches generated from two spatially separated annotation nodes as negative training samples. We used $\sim 919 \mathrm{~K}$ training pairs, $460 \mathrm{~K}$ of them being positive pairs and $\sim 459 \mathrm{~K}$ being negative pairs.

In connection (Fig. 4c), a $1 \times M$ feature vector is extracted from individual input patch. (M can be defined by the user, and we used $M=200$ in our experiment.) The Euclidean distance between two feature vectors is calculated as the dissimilarity score of a patch pair, which is multiplied by the distance to form the weight in our proposed DMST graph.
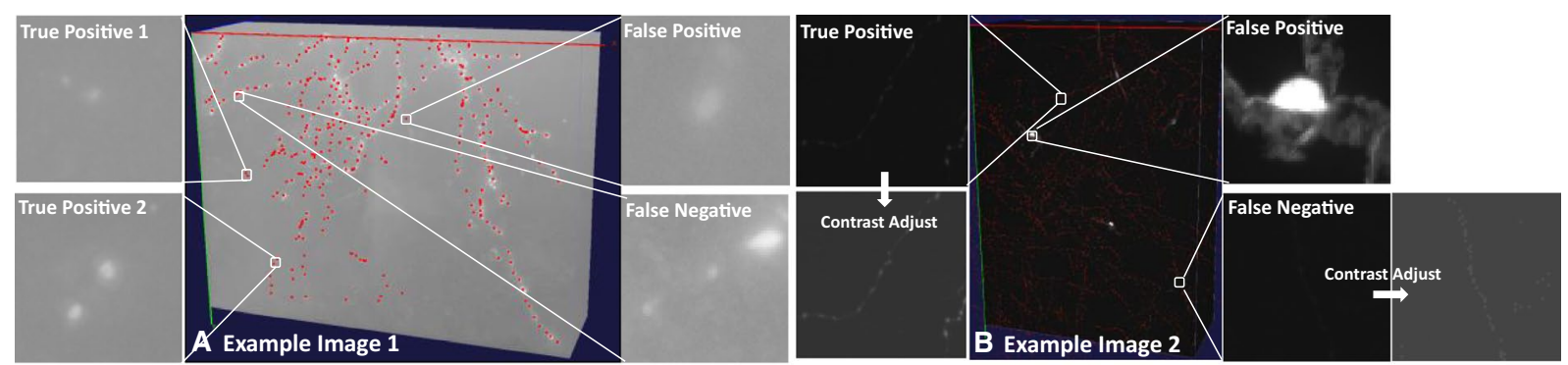

Fig. 3 Axon detection results on two challenging datasets. (a) An example of a 3D image stack of mouse neuron imaged with bright-field microscopy (also see Fig. 2a). (b) An example of a 3D stack (shown as cropped) from a whole mouse brain imaged with fMOST; the pixel resolution is $0.3 \mathrm{um} \times 0.3 \mathrm{um} \times 1 \mathrm{um}$. Red dots indicate detected axonal signals. The two false positive example patches shown in (a) and (b) could be eliminated with more training samples. Those false negative patches (also as shown in (a) and (b)) with very weak signals at the center could be further identified by increasing the amount of weak signal foreground samples in the training set 


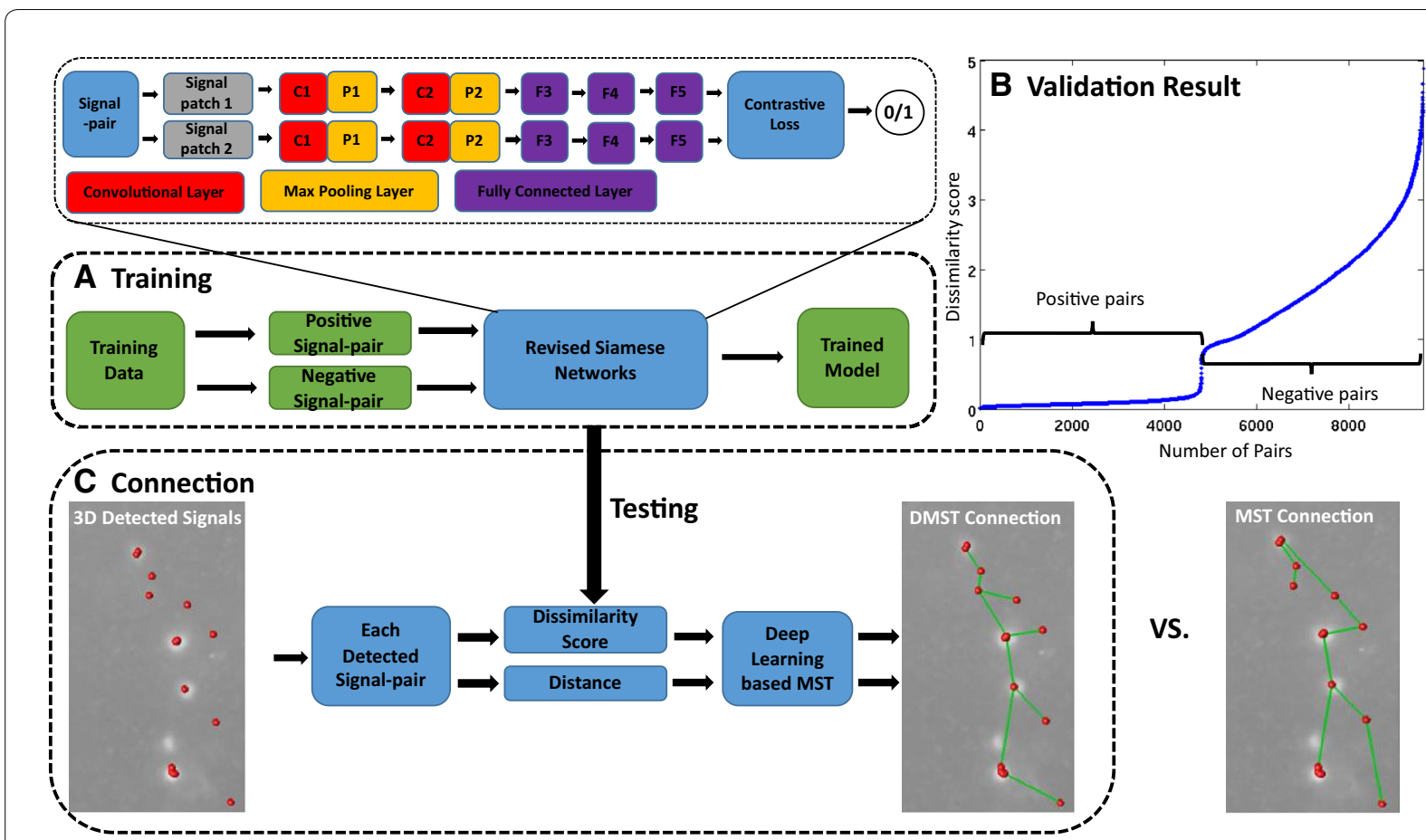

Fig. 4 The workflow of neurite connection module. (a) In the training step, the connectivity of a signal pair is learned using a revised Siamese network. In each pair, two $1 \times 200$ feature vectors are extracted. (b) The validated result with sorted dissimilarity scores for all signal pairs shows that the dissimilarity scores of positive pairs are much lower than that of negative pairs. (c) The trained model is applied in the connection step to calculate the dissimilarity for each detected signal pair. Results of our DMST connection and the original MST connection (using distance as the weight only) are shown
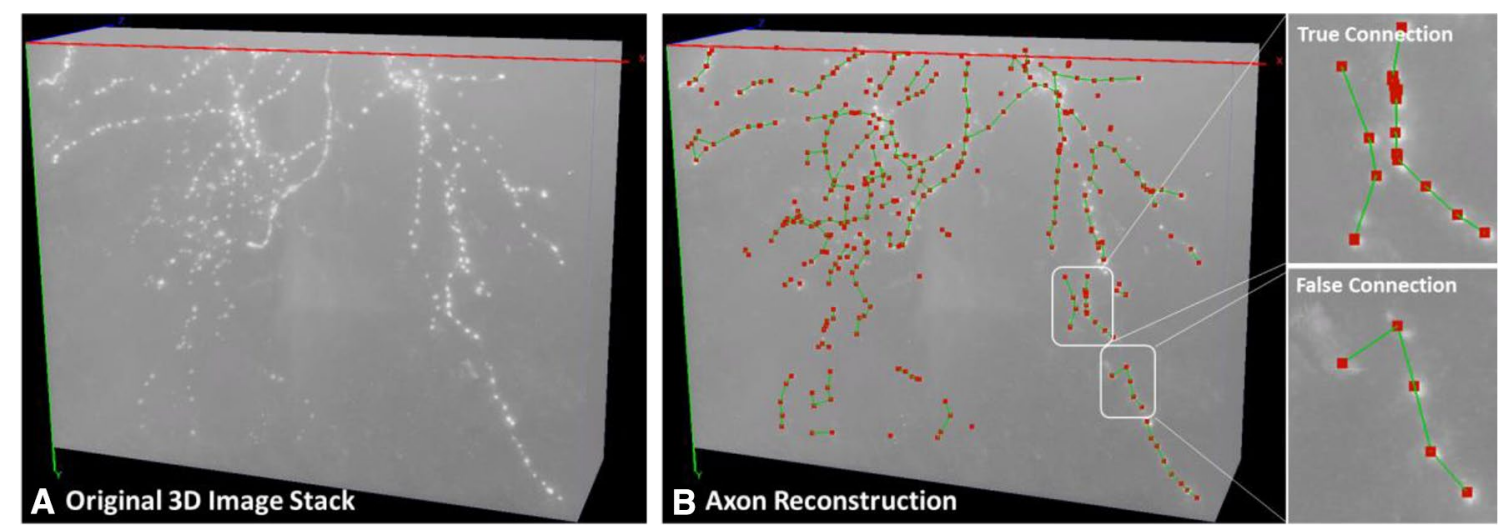

Fig. 5 DeepNeuron axon reconstruction. (a) The same example image as shown in Fig. 2a. (b) 3D axon signals (red dots) were extracted with neurite signal detection module; local 3D connections (green lines) between signals were generated by neurite connection module. As shown in B, the proposed DMST in the connection module can formulate the correct neurite structure even when the detected signals from different neurites are spatially close to one another. In a more difficult case, however, the loss of true signal in the local area can make the information captured by the network outweighed by the distance, resulting in false connection. This could be avoided by adequately enlarging the examined local area. Note that unconnected fragments like those on the bottom left would be further processed by our non-deep-learning functions of the tool, which is out of the scope of this article 
Combing neurite signal detection and neurite connection modules, we were able to reconstruct axons that present big challenges to traditional methods due to large gaps between signal segments (Fig. 5).

\subsection{Smart pruning}

Many of the existing automatic tracing algorithms rely on the correct estimation of the threshold that separates the potential foreground signal from the background. Typical methods include those that use the weighted average intensity of the entire image to threshold the image or add a preprocessing step to enhance signals. These methods have limited success for neuron images with low signal-to-noise ratio and uneven background. To solve the problem, we developed a smart pruning module in DeepNeuron. It relieves the burden of precisely separating foreground and background up the front. Using existing algorithms, our module first generated over-traced results with a lower foreground/background segregation threshold or through signal enhancement step. We then trained CNN networks to classify true signals and false positive signals. Using the trained models, we filtered out falsely detected signals and pruned the reconstructed neuronal tree. Furthermore, different tracing results generated from multiple base tracing algorithms could be combined [23] to produce a consensus using this module (Fig. 6).

\subsection{Manual reconstruction evaluation}

Since manual reconstruction is largely used as the gold standard to evaluate automated reconstruction algorithms and to generate training set for machine learning-based approaches, it is important to assess the consistency of manual reconstructions among different annotators or of the same annotator at different times. For this purpose, DeepNeuron provides an evaluation module based on deep learning classification model. Take the mouse neuron dataset from the Allen Cell Type Database [24] we described in Sect. 2.1 as an example, we divided the 122 manual reconstructions from multiple annotators into five subsets and took a fivefold crossvalidation strategy. Each time we took four subsets as the training data. Once the network was trained, we used it to evaluate how consistent the remaining subset is with respect to the training subsets (Fig. 7, Table 2). More specifically:
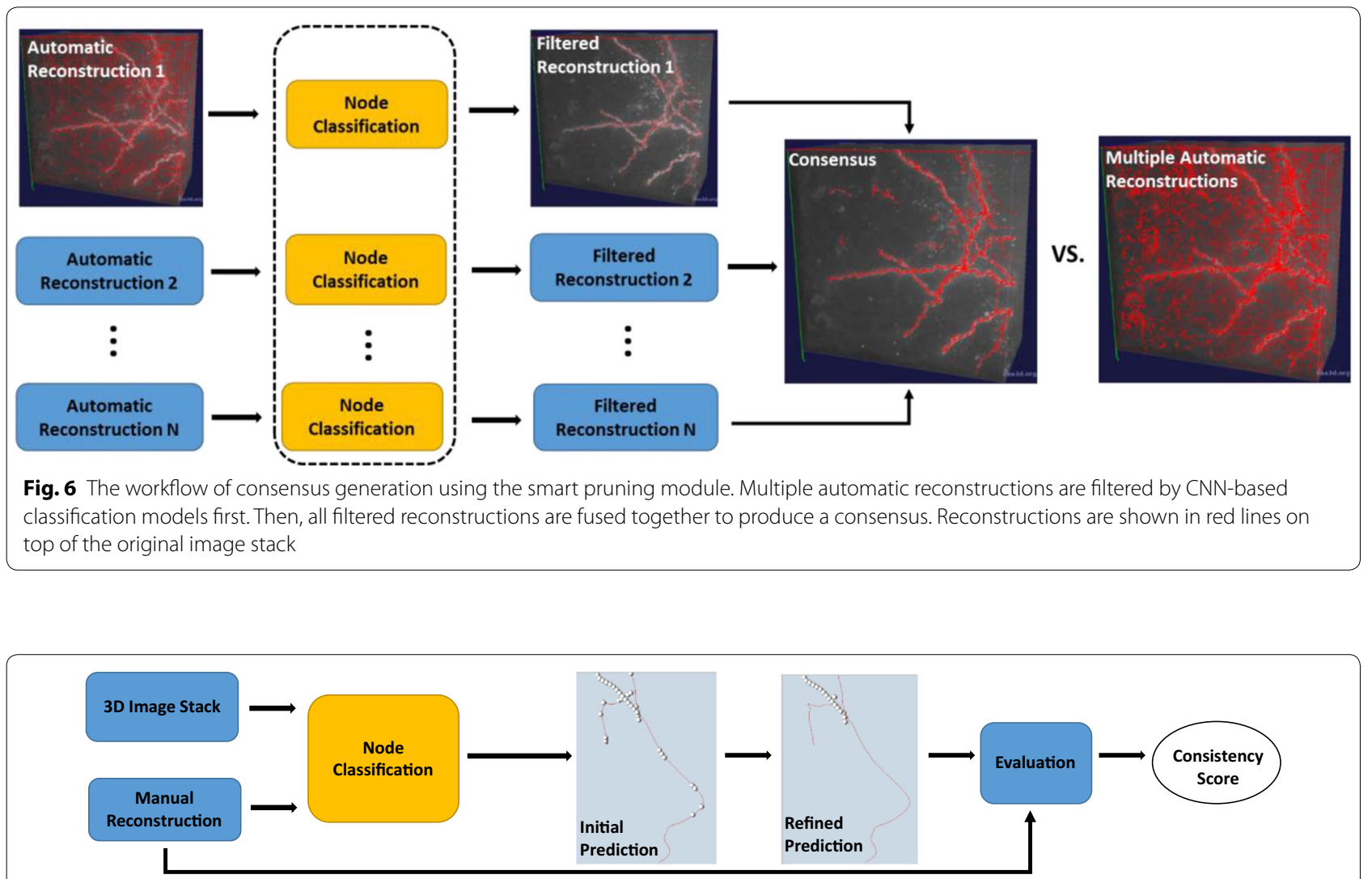

Fig. 7 The workflow of manual reconstruction evaluation module. All annotation nodes are classified into foreground (red lines) and background (white dots). Gaps in the initial prediction were automatically filled based on the orientation, tip location, and distance in the refined prediction 
Table 2 Fivefold cross-validation on 122 manual reconstructions of biocytin-labeled mouse neuron dataset

\begin{tabular}{|c|c|c|c|c|c|c|}
\hline \multirow[t]{3}{*}{ Training set } & \multicolumn{6}{|c|}{ Consistency score } \\
\hline & \multicolumn{3}{|l|}{ Training } & \multicolumn{3}{|l|}{ Validation } \\
\hline & Axon (\%) & Dendrite (\%) & Overall (\%) & Axon (\%) & Dendrite (\%) & Overall (\%) \\
\hline$\{1-122\} \backslash\{1-24\}$ & 97.02 & 99.35 & 98.45 & 95.90 & 99.74 & 98.30 \\
\hline$\{1-122\} \backslash\{25-48\}$ & 96.69 & 99.68 & 98.45 & 97.51 & 98.07 & 97.94 \\
\hline$\{1-122\} \backslash\{49-72\}$ & 97.14 & 99.31 & 98.52 & 95.66 & 99.79 & 97.95 \\
\hline$\{1-122\} \backslash\{73-96\}$ & 96.67 & 99.34 & 98.41 & 96.48 & 99.58 & 98.02 \\
\hline$\{1-122\} \backslash\{97-122\}$ & 96.82 & 99.39 & 98.36 & 98.07 & 99.61 & 99.15 \\
\hline Average & 96.87 & 99.41 & 98.45 & 96.47 & 99.34 & 98.25 \\
\hline
\end{tabular}

Table 3 Comparison results of consistency scores on human and mouse neuron reconstructions

\begin{tabular}{lllll}
\hline & Number & Axon (\%) & Dendrite (\%) & Overall (\%) \\
\hline Human & 10 & 98.23 & 99.60 & 98.93 \\
Mouse & 21 & 94.28 & 99.40 & 95.38 \\
\hline
\end{tabular}

- First, all annotation nodes in test subset were classified into two categories: foreground and background.

- All classified foreground nodes formed an initial prediction.

- Based on the orientation, tip location, and distance, fragments in the initial prediction were automatically connected to produce a refined prediction. In our experiment, we only connected terminal tips between two segments whose orientation differs less than 30 degrees and distance is smaller than 30 voxels.

- The test subset is evaluated by the consistency score $c$ :

$$
c=\frac{\text { Number of nodes in the refined prediction }}{\text { Number of nodes in the manual reconstruction }} \times 100 \%
$$

Table 2 shows the fivefold cross-validation results on 122 manual reconstructions for the bright-field biocytin-labeled mouse neuron dataset from Allen Cell Type Database [24]. The high consistency scores indicate that manual reconstructions are very consistent across different annotators and different subsets of data. In addition, thicker and more continuous dendrites $(>99 \%)$ have higher consistency scores than dim and discontinuous axons ( $>96 \%$ ), which are harder to reconstruct.

More broadly, we applied our evaluation module to 31 manual reconstructions including 10 human neurons and 21 mouse neurons in the Allen Cell Type Database [24].
Table 3 shows our comparison results. Consistent with Table 2, dendrites have higher scores than axons. In addition, scores on human neurite (axon and dendrite) reconstructions are higher than those of mouse, indicating that annotators have better tracing performance on physically larger human neurons.

\subsection{Classification of dendrites and axons}

Dendrites and axons have their own functions and play different roles in the nervous system. Distinguishing these two types of neurites can help us gain insight into the brain circuitry. Although dendrites and axons show different shapes and intensity properties in light microscopy images, such a general rule of thumb, however, is not always guaranteed. Due to variant image quality, axons can also appear continuous and look more like dendrites. This makes them difficult to be correctly labeled in most of tracing algorithms. Here we present a deep learning module serving as a vehicle for the networks that are trained for this purpose. This tool allows to automatically classify dendrites and axons on realtime manual annotation, and potentially save time for annotators.

We used the same approach as described in Sect. 2.1, except that the problem is now a multinomial classification (dendrite, axon, and background) instead of a binary classification (foreground and background) problem. Figure 8 shows the performance on two testing cases from the Allen Cell Type Database [24]. In this example, we used $\sim 813 \mathrm{~K}$ training samples including $\sim 143 \mathrm{~K}$ axons, $\sim 261 \mathrm{~K}$ dendrites, and $\sim 409 \mathrm{~K}$ background. In this article, AlexNet and a revised model were used as demonstration. In Fig. 8, both AlexNet and our revised model can accurately classify continuous dendritic and discrete axonal signals. However, when the signal of axons is similar to dendrites (Fig. 8b), AlexNet mistakenly classified axons into dendrites, while the revised model with one more convolutional layer successfully distinguished axons from 

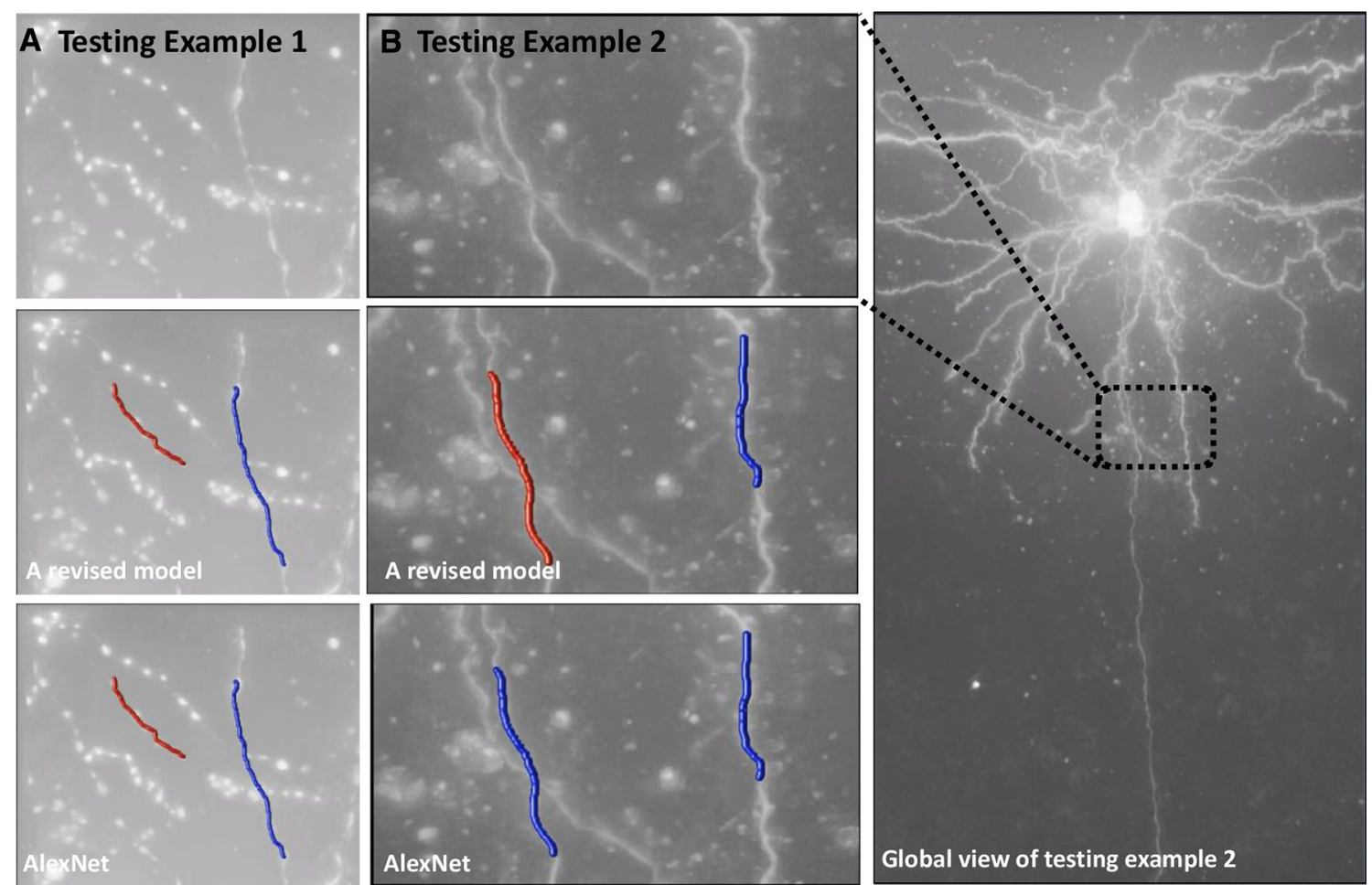

Fig. 8 Comparison of dendrite and axon classification using AlexNet and a revised model. (a) Axons are discrete, and dendrites are continuous. (b) Both axons and dendrites are continuous. The left segment is extracted from a long axon. The right segment is extracted from a local dendrite. Red color indicates the axon, and blue color indicates the dendrite in $(\mathbf{a})$ and $(\mathbf{b})$. Note that all these 3D segments are manually annotated using Virtual Finger technology [7, 9]; neurite types are automatically annotated by the proposed module

Table 4 Comparison of AlexNet with a revised model

\begin{tabular}{lclll}
\hline $\begin{array}{l}\text { Deep learning } \\
\text { network }\end{array}$ & $\begin{array}{l}\text { Averaged } \\
\text { forward- } \\
\text { backward } \\
\text { time (s) }\end{array}$ & $\begin{array}{l}\text { Deemed } \\
\text { axon } \\
\text { (\%) }\end{array}$ & $\begin{array}{l}\text { Deemed } \\
\text { dendrite } \\
\text { (\%) }\end{array}$ & $\begin{array}{l}\text { Deemed } \\
\text { background } \\
\text { (\%) }\end{array}$ \\
\hline AlexNet & 3.27 & 84.79 & 93.86 & 99.04 \\
$\begin{array}{c}\text { A revised } \\
\text { model }\end{array}$ & 335.91 & 97.89 & 98.55 & 99.82 \\
\hline
\end{tabular}

dendrites. Table 4 shows the comparison of classification accuracy between the two models on the training samples. We found that the revised model yields much better classification performance. In exchange, the efficiency of the revised model is sacrificed due to much more number of outputs in each convolutional layer (3.27-s forward-backward time for AlexNet; 335.91-s forward-backward time for the revised model).

\section{Discussion}

In this paper, we presented a new deep learningbased Open Source toolbox for neuron tracing: DeepNeuron. With extensible framework, DeepNeuron currently provides five modules to comprehend the major tasks:

- For a neuron image stack, it can be used to automatically detect neurite signals.

- For a neuron image stack with detected 3D signals, it can automatically connect signals to generate local segments.

- For a neuron image stack with its associated automated reconstruction, it can be used as a filter to clean up all false positive tracing and generate a refined result.

- For a neuron image stack with its associated manual reconstructions, it can evaluate how consistent and reliable the reconstructions are.

- For a neuron image stack with interactive human annotation via the user interface, it can label neurite types in real time. 
DeepNeuron has been implemented as an Open Source plugin in Vaa3D (http://vaa3d.org) [7, 8]. DeepNeuron toolbox is a highly flexible vehicle allowing investigators to take advantage of deep learning to facilitate neuron tracing in their research. As mentioned in this article, researchers can freely replace different network models that suit their needs. Combined with other related features in Vaa3D including 30+ automatic neuron tracing plugins, semiautomatic neuron annotation, annotation utilities, neuron image/reconstruction visualization, DeepNeuron works as a smart artificial intelligence engine which offers great help to biologists in exploring neuronal morphology.

\section{Toolbox and software availability}

The DeepNeuron toolbox was written in $\mathrm{C}++$ as a plugin to Vaa3D. DeepNeuron source code is available at https:// github.com/Vaa3D/vaa3d_tools/tree/master/hackathon/ MK/DeepNeuron. In addition, the DeepNeuron plugin is also included as a plugin in binary releases of Vaa3D, which can be downloaded at https://github.com/Vaa3D/ Vaa3D_Data/releases/tag/1.0.

\section{Authors' contributions}

HP conceived and managed the project. FL proposed the overall technical framework. ZZ developed the toolbox and conducted the experiments. HK implemented a plugin for the dendrite and axon classification and assisted in several other experiments. All authors edited the manuscript. All authors read and approved the final manuscript

\section{Author details}

${ }^{1}$ Allen Institute for Brain Science, Seattle, USA. ${ }^{2}$ Southeast University - Allen Institute Joint Center for Neuron Morphology, Southeast University, Nanjing, China.

\section{Acknowledgements}

We thank Allen Institute for Brain Science for providing neuron datasets and manual annotations. The authors wish to thank the Allen Institute founders, $P$. G. Allen and J. Allen, for their vision, encouragement, and support.

\section{Competing interests}

On behalf of all authors, the corresponding author states that there is no competing interests.

\section{Ethics approval and consent to participate} Not applicable.

\section{Publisher's Note}

Springer Nature remains neutral with regard to jurisdictional claims in published maps and institutional affiliations.

Received: 26 February 2018 Accepted: 18 April 2018

Published online: 06 June 2018

\section{References}

1. Choromanska A, Chang S-F, Yuste R (2012) Automatic reconstruction of neural morphologies with multi-scale tracking. Front Neural Circuits 6:25
2. Donohue DE, Ascoli GA (2011) Automated reconstruction of neuronal morphology: an overview. Brain Res Rev 67:94-102

3. Feng $L$, Zhao T, Kim J (2015) neuTube 1.0: a new design for efficient neuron reconstruction software based on the SWC format. eNeuro 2:ENEURO-0049

4. Longair MH, Baker DA, Armstrong JD (2011) Simple neurite tracer: open source software for reconstruction, visualization and analysis of neuronal processes. Bioinformatics 27:2453-2454

5. Luisi J, Narayanaswamy A, Galbreath Z, Roysam B (2011) The FARSIGHT trace editor: an open source tool for 3-D inspection and efficient pattern analysis aided editing of automated neuronal reconstructions. Neuroinformatics 9:305-315

6. Meijering E, Jacob M, Sarria JC, Steiner P, Hirling H, Unser M (2004) Design and validation of a tool for neurite tracing and analysis in fluorescence microscopy images. Cytom Part A 58:167-176

7. Peng H, Bria A, Zhou Z, lannello G, Long F (2014) Extensible visualization and analysis for multidimensional images using Vaa3D. Nat Protoc 9:193-208

8. Peng H, Ruan Z, Long F, Simpson JH, Myers EW (2010) V3D enables realtime 3D visualization and quantitative analysis of large-scale biological image data sets. Nat Biotechnol 28:348-353

9. Peng H, Tang J, Xiao H, Bria A, Zhou J, Butler V, Zhou Z, Gonzalez-Bellido PT, Oh SW, Chen J, Mitra A, Tsien RW, Zeng H, Ascoli GA, lannello G, Hawrylycz M, Myers E, Long F (2014) Virtual finger boosts three-dimensional imaging and microsurgery as well as terabyte volume image visualization and analysis. Nat Commun 5:4342

10. Liu Y (2011) The DIADEM and beyond. Neuroinformatics 9:99-102

11. Peng H, Hawrylycz M, Roskams J, Hill S, Spruston N, Meijering E, Ascoli GA (2015) BigNeuron: large-scale 3D neuron reconstruction from optical microscopy images. Neuron 87:252-256

12. Peng H, Zhou Z, Meijering E, Zhao T, Ascoli GA, Hawrylycz M (2017) Automatic tracing of ultra-volumes of neuronal images. Nat Methods 14:332

13. LeCun Y, Bengio Y, Hinton G (2015) Deep learning. Nature 521:436

14. Gala R, Chapeton J, Jitesh J, Bhavsar C, Stepanyants A (2014) Active learning of neuron morphology for accurate automated tracing of neurites. Front Neuroanat 8:37

15. Chen H, Xiao H, Liu T, Peng H (2015) SmartTracing: self-learning-based neuron reconstruction. Brain Inform 2:135-144

16. Fakhry A, Peng H, Ji S (2016) Deep models for brain EM image segmentation: novel insights and improved performance. Bioinformatics $32: 2352-2358$

17. Li R, Zeng T, Ji S (2017) Deep learning segmentation of optical microscopy images improves 3D neuron reconstruction. IEEE Trans Med Imaging 36:1533-1541

18. Krizhevsky A, Sutskever I, Hinton GE (2012) Imagenet classification with deep convolutional neural networks. In: Advances in neural information processing systems, pp 1097-1105

19. Cheng Y (1995) Mean shift, mode seeking, and clustering. IEEE Trans Pattern Anal Mach Intell 17:790-799

20. Gong H, Xu D, Yuan J, Li X, Guo C, Peng J, Li Y, Schwarz LA, Li A, Hu B (2016) High-throughput dual-colour precision imaging for brain-wide connectome with cytoarchitectonic landmarks at the cellular level. Nat Commun 7:12142

21. Bromley J, Guyon I, LeCun Y, Säckinger E, Shah R (1994) Signature verification using a" siamese" time delay neural network. In: Advances in neural information processing systems, pp 737-744

22. Chopra S, Hadsell R, LeCun Y (2005) Learning a similarity metric discriminatively, with application to face verification. In: IEEE computer society conference on computer vision and pattern recognition. CVPR 2005. IEEE, pp 539-546

23. Wan Y, Long F, Qu L, Xiao H, Hawrylycz M, Myers EW, Peng H (2015) BlastNeuron for automated comparison, retrieval and clustering of 3D neuron morphologies. Neuroinformatics. https://doi.org/10.1007/s12021-12015 $-19272-12027$

24. Allen Institute for Brain Science (2015) Allen cell types database. http:// celltypes.brain-map.org/. Accessed Jan 2018 